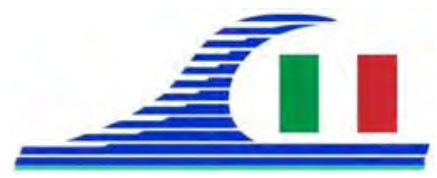

Conférence Méditerranéenne Côtière et Maritime EDITION 3, FERRARA, ITALIA (2015)

Coastal and Maritime Mediterranean Conference

Disponible en ligne - http://www.paralia.fr - Available online

\title{
Systèmes de protection contre les submersions marines Retour d'expérience Xynthia. Conclusions des études de cas
}

\author{
Marc IGIGABEL ${ }^{1}$, Yves NEDELEC ${ }^{2}$, Nicolas FLOUEST ${ }^{2}$, \\ Nathalie BERENGER ${ }^{3}$, Patrick CHASSE ${ }^{1}$, Alexis BERNARD ${ }^{3}$, Christian PITIE $^{4}$ \\ 1. Cerema, Direction technique Eau, Mer et Fleuves, France. \\ \{marc.igigabel ; patrick.chasse\}@cerema.fr \\ 2. Cerema, Direction territoriale Sud-Ouest, France \\ \{yves.nedelec; nicolas.flouest\}@cerema.fr \\ 3. Cerema, Direction territoriale Ouest, France. \\ \{nathalie.berenger; alexis.bernard\}@cerema.fr \\ 4. Conseil Général de l’Environnement et du Développement Durable, France. \\ christian.pitie@developpement-durable.gouv.fr
}

\section{Résumé :}

La définition des frontières et de la structure interne d'un système de protection contre les submersions marines, puis la compréhension du comportement du système face aux événements passés contribuent largement à déterminer les actions qui peuvent être engagées pour améliorer sa performance. Afin d'établir dans ce domaine une méthodologie éprouvée et transposable à d'autres territoires frappés par des épisodes de submersions marines, une réflexion a été engagée sur des territoires touchés par la tempête Xynthia en février 2010. Trois sites de Charente-Maritime et un site de LoireAtlantique ont été retenus pour les configurations contrastées qu’ils présentent :

- Loix est une presqu'île entourée par un ensemble de bassins et de marais,

- le site des Boucholeurs s'étend en bordure d'une vaste baie. En front de mer des habitations sont directement exposées aux vagues, tandis qu'en retrait des constructions occupent l'emplacement d'anciens marais endigués,

- le site de Boyardville-La Perrotine est une zone urbanisée traversée par un canal endigué reliant le front de mer à une vaste zone de marais et desservant un port de plaisance,

- Batz-sur-Mer se caractérise par des marais salants en bordure desquels l’urbanisation s’est développée.

Ces sites sont structurés par des formations naturelles, par des digues, des canaux et des fossés, des infrastructures routières et ferroviaires, impliquant dans leur gestion des acteurs multiples. Ces sites sont représentatifs de la plupart des situations rencontrées au cours de Xynthia. L'article proposé présente les conclusions des études de cas en se focalisant sur la manière dont les enjeux principaux identifiables étaient effectivement protégés lors du passage de la tempête.

Mots-clés : Inondation, Submersion, Protection, Système, Xynthia. 
Côtes méditerranéennes menacées :

Risques et défis dans le contexte du changement climatique

\section{Introduction}

Quatre sites touchés par Xynthia (Loix, Les Boucholeurs et Boyardville en CharenteMaritime et Batz-sur-mer en Loire Atlantique) ont fait l'objet d'une analyse de leurs systèmes de protection en prenant comme point focal le secteur aux plus forts enjeux (IGIGABEL et al., sous presse). Cet article présente les conclusions des études de cas.

\section{Loix}

Le site de Loix se caractérise par un bourg entouré par un ensemble de bassins et de marais. L'analyse est centrée sur le quartier de Lavaud positionné en arrière des bassins. Les casiers forment un cordon le long de la façade maritime. Si l'on considère l'organisation du site vis-à-vis de la protection du quartier Lavaud, il apparaît un schéma en cascade : lorsque les casiers sont remplis et débordent, les entrées d'eau des casiers 1, 2 et 3 sont susceptibles de s'écouler dans un mouvement d'ensemble vers le casier 4 qui lui-même peut recevoir directement des entrées d'eau du front de mer. Les écoulements peuvent se produire d'un casier à un autre par les digues de séparation ou par la zone arrière comprise entre les casiers et la ligne abritée (cf. figure 1).

Au cours de Xynthia, le quartier Lavaud a subi une submersion très rapide : tandis que les bassins environnants se remplissaient, de multiples brèches se formaient sur la digue maritime. Dans ces circonstances, peu avant le pic de marée, les écoulements se sont orientés de façon quasi-synchrone et avec de très forts débits vers la zone basse occupée par le quartier Lavaud.

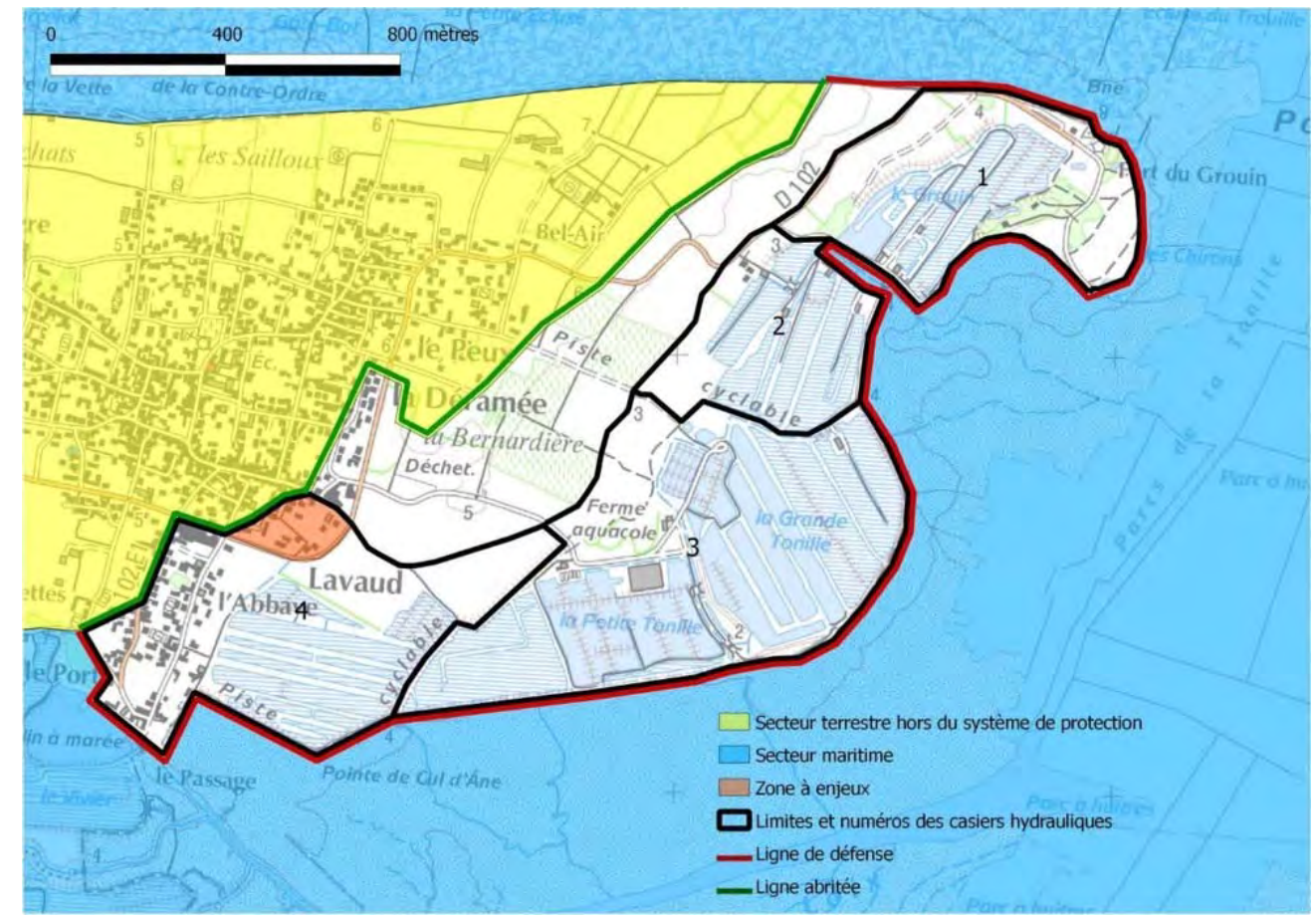

Figure 1. Système de protection de Loix. 


\section{Les Boucholeurs}

Le site des Boucholeurs est une zone étendue et assez densément urbanisée. L'analyse est centrée sur la zone habitée des Boucholeurs (cf. figure 2). Au cours de Xynthia, les défauts de protection suivants sont apparus :

- $\quad$ au nord, les ouvrages du front de mer n’ont pas protégé suffisamment contre les franchissements. Le déferlement des vagues est à l’origine de deux décès.

- $\quad$ au sud, le cordon littoral était très fragile et les capacités de stockage du marais ont été largement dépassées.

A marée montante, seules les eaux du casier 2 ont pu rejoindre le casier 1 . Si la voie ferrée et la voie express ont partiellement orienté les eaux du casier 2 vers la zone à enjeux, elles ont aussi laissé une partie des eaux s'étendre dans le casier 3. Les eaux envahissant le casier 4 ont débordé dans le casier 3 sans atteindre les casiers 1 et 2 .

Le canal de Port Punay qui traverse le secteur urbanisé a favorisé l'inondation en orientant les écoulements vers ce secteur. Si son faible débit entrant n'a pas contribué significativement à la submersion, il n’a pas permis non plus une évacuation rapide, ce qui a entraîné une submersion préjudiciable sur plusieurs jours.

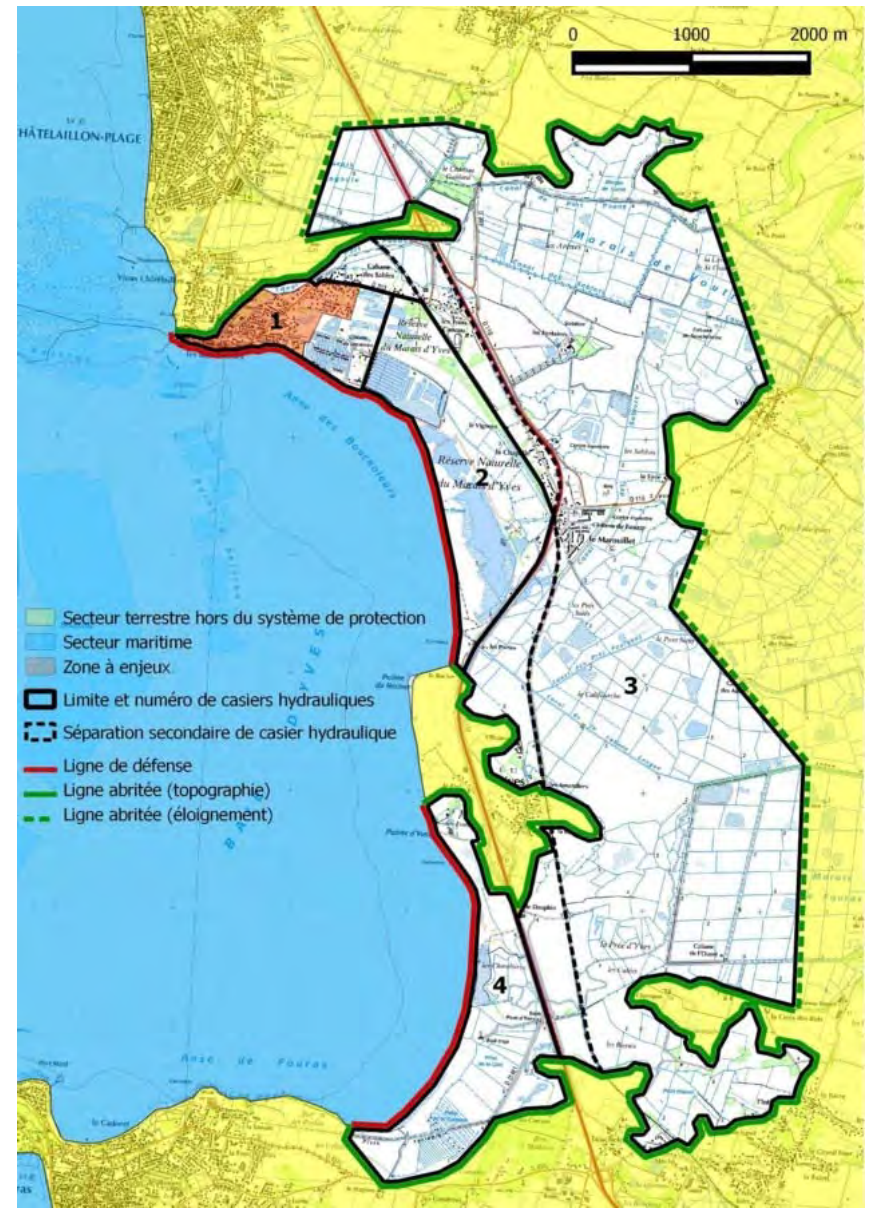

Figure 2. Système de protection des Boucholeurs. 
Côtes méditerranéennes menacées :

Risques et défis dans le contexte du changement climatique

\section{Boyardville}

Le site de Boyardville-La Perrotine est une zone urbanisée traversée par un chenal endigué navigable reliant le front de mer à une vaste zone de marais. Ce site est analysé en ne considérant qu'un seul système de protection, le chenal permettant les échanges hydrauliques, d'une part entre le front de mer et le marais, et d'autre part entre ses deux rives. L'analyse est centrée sur le quartier de Boyardville (cf. figure 3).

Les chemins hydrauliques potentiels conduisant au casier 1 (Boyardville) sont :

- $\quad$ entre le chenal dans sa partie aval et Boyardville (chemin le plus direct),

- $\quad$ entre le marais et Boyardville, du casier 3 vers le casier 1 (par l'ouest),

- $\quad$ entre le marais et Boyardville, du casier 3 vers le casier 1, en passant par le casier 4 (contournement large par le nord).

Au cours de Xynthia, du fait du niveau marin exceptionnel, le fonctionnement du chenal a été perturbé : peu de temps avant le pic de marée, une arrivée d’eau supplémentaire en provenance du polder submergé de la Perrotine s'est additionnée à l'écoulement normal. Les principales entrées d'eau dans le casier 1 se sont produites en aval du canal où la perte de charge était la moins importante et où les écoulements en provenance de la Perrotine débouchaient. L'inondation de la cuvette de Boyardville, bien que partielle (hauteur d'eau comprises entre 1,40 et 1,65 m), a causé un décès. Un niveau marin supérieur de 0,15 m aurait engendré des hauteurs d'eau comprises entre 1,75 et 2,00 m.

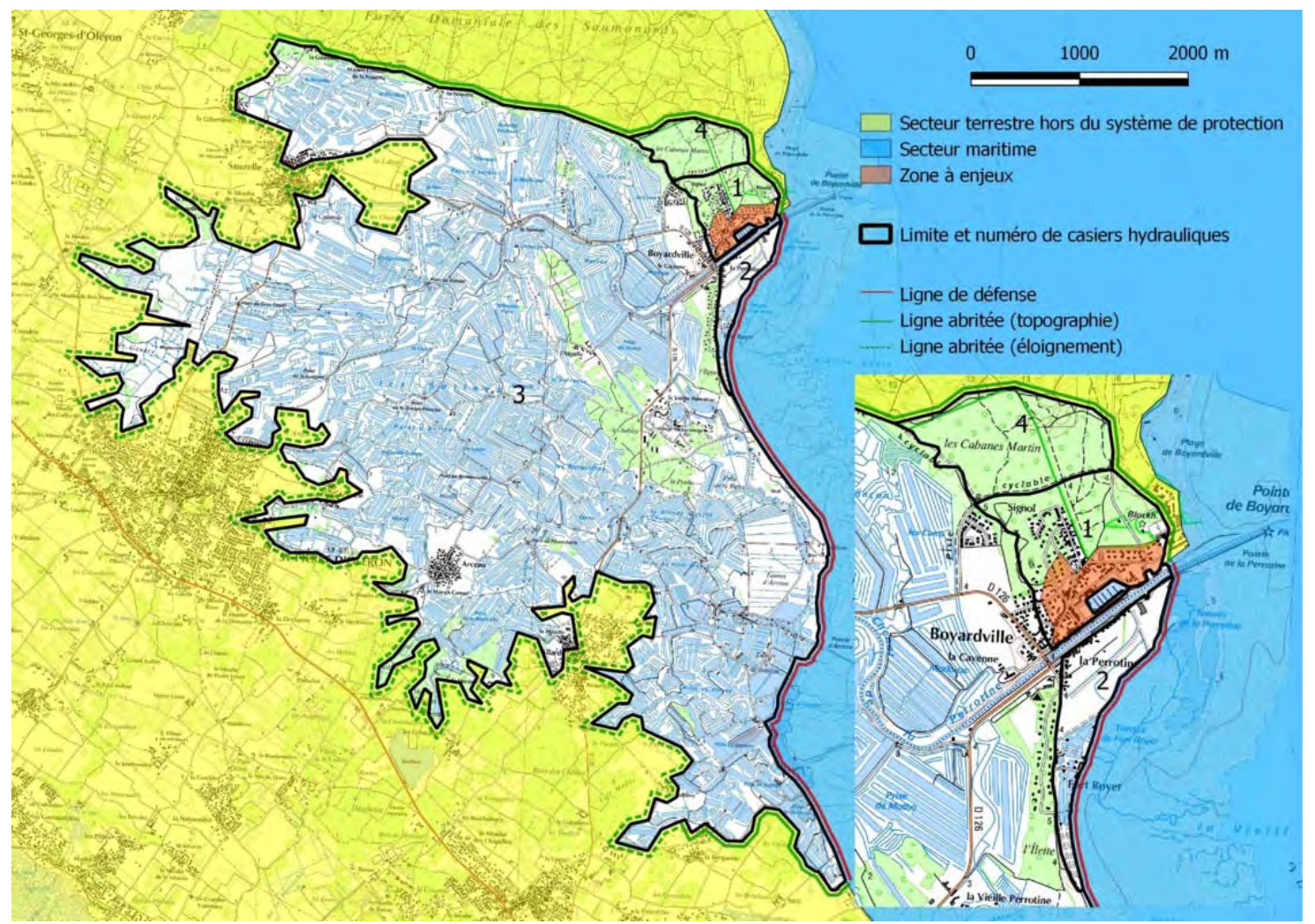

Figure 3. Système de protection de Boyardville. 
Mediterranean coasts at threat:

Hazards and challenges in the context of climate variability

\section{Batz-sur-Mer}

Le site de Batz-sur-Mer (figure 4) se caractérise par de grands espaces de marais salants en bordure desquels l'urbanisation s'est développée. L'analyse est centrée sur le quartier d'habitation de la Herpe.

L'étude menée met en évidence un système constitué de :

- $\quad$ quatre casiers alignés sur le front de mer et séparés par des étiers,

- $\quad$ une zone arrière dans laquelle est situé le quartier de la Herpe.

Cette position du quartier de la Herpe en retrait des casiers résulte du fait que son altitude est supérieure aux points bas de la ligne de défense constituée par la digue maritime. Cette structure n'exerce donc pas d'action de retenue de l'eau vis-à-vis de ce quartier, ce qui le met à l'abri d'effets d'inondations rapides par rupture de digue ou surverse. Par ailleurs, le remplissage des bassins est suffisamment rapide pour que l'on puisse considérer que le système ne retarde pas non plus la propagation de l'inondation au niveau de cette zone d'enjeux. Les étiers, bien que certains soient équipés de barrages, constituent des voies privilégiées d'écoulement.

Les digues et bassins ont donc un effet neutre sur les niveaux d'eau qui apparaissent sur le quartier de la Herpe. Par contre ce dispositif conserve un rôle positif en atténuant fortement la propagation des vagues.

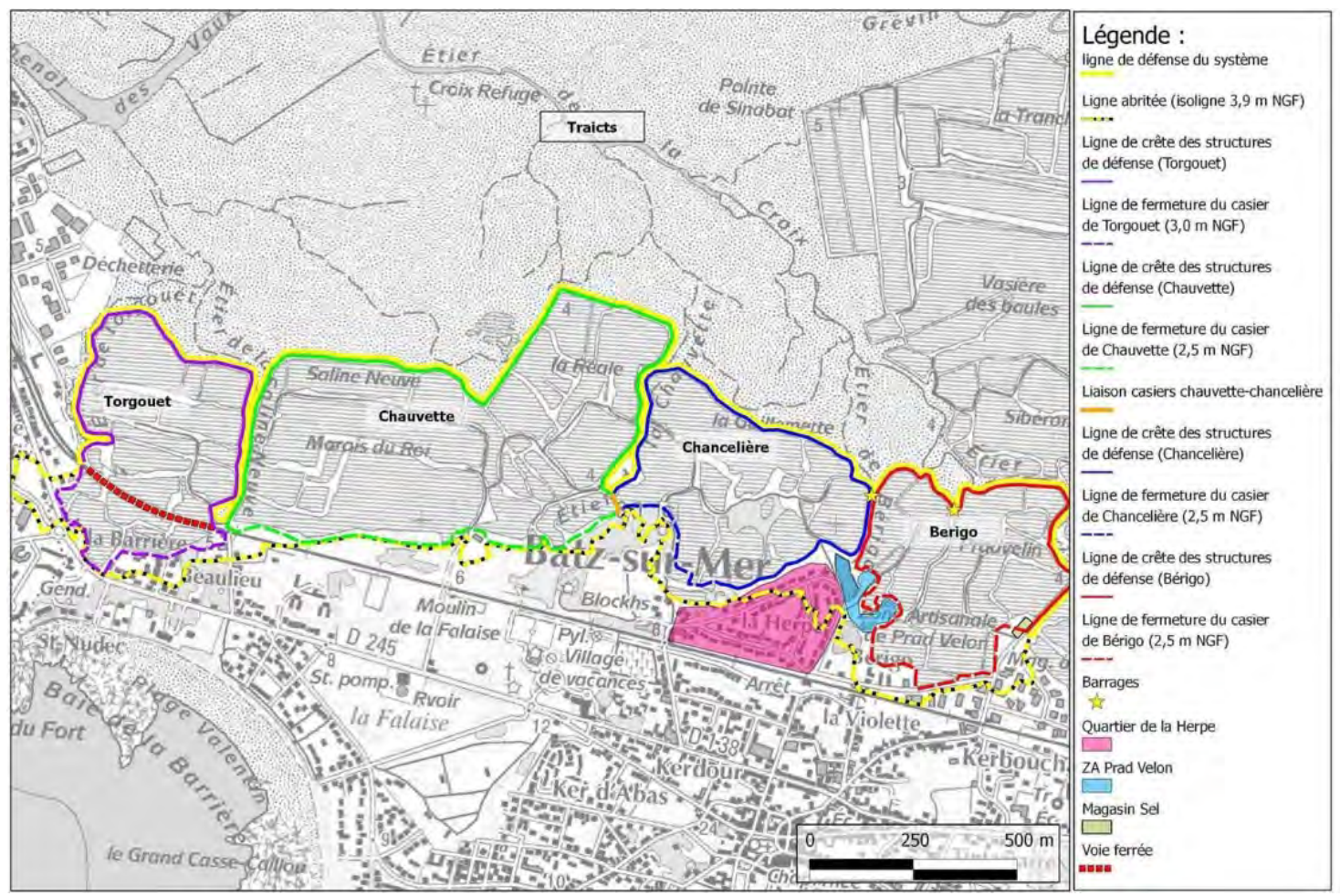

Figure 4. Système de protection de Batz-sur-Mer. 
Côtes méditerranéennes menacées :

Risques et défis dans le contexte du changement climatique

\section{Conclusion}

Les sites étudiés présentent des configurations contrastées qui ont engendré au cours de Xynthia différents scénarios de submersion :

- $\quad$ sur le site de Loix, le quartier Lavaud occupe une position singulière : éloignée du front de mer, il se situe potentiellement à la jonction des flux en provenance des différents casiers. Les brèches et la saturation des bassins tampons ont provoqué une submersion très rapide de ce quartier peu avant le pic de marée.

- $\quad$ sur le site des Boucholeurs, la zone urbanisée a subi à la fois des franchissements sur son front de mer (exposition forte aux vagues à l'origine des deux décès) et des arrivées d'eau générées par les débordements de digues et cordons dunaires sur des linéaires très importants au sud. Le canal qui traverse la zone urbanisée a contribué à accumuler l'eau dans la zone aux plus forts enjeux. La perméabilité des infrastructures ferroviaires et routières ont cependant permis un certain étalement de l'eau hors des zones les plus sensibles.

- $\quad$ sur le site de Boyardville-La Perrotine, l'inondation de la cuvette de Boyardville dépend du niveau du canal qui traverse le site. En plus de son alimentation normale depuis le front de mer, le canal a reçu des eaux traversant la Perrotine. Bien que partielle, l'inondation de la cuvette a provoqué un décès.

- $\quad$ Sur le site de Batz-sur-Mer, le quartier de la Herpe est séparé du front de mer par une série de bassins. Ce quartier est positionné à une altitude plus élevée que la crête de la digue maritime, ce qui le met à l'abri d'une submersion rapide.

Les configurations les plus dangereuses sont celles qui engendrent des submersions rapides. Certaines sont faciles à identifier : cuvettes positionnées immédiatement en arrière d'une digue (Boyardville, dont la situation est comparable à la Faute-sur-Mer en Vendée où la tempête Xynthia a provoqué 29 décès) ou secteurs exposés aux vagues (front de mer des Boucholeurs). D’autres sont plus difficiles à déceler: cuvettes positionnées en arrière de bassins tampons (cas du quartier Lavaud à Loix) ou zones basses traversées par un canal non endigué (cas des Boucholeurs). L'éloignement de la côte ne met donc pas nécessairement à l'abri des phénomènes de submersion rapide.

Enfin, certaines zones, bien qu'inondables ne présentent que peu de risque pour la vie de leurs occupants grâce à leurs situations hors zones d'influence des digues et hors cuvettes (cas du quartier de la Herpe à Batz-sur-Mer). En cas de submersion, la montée des eaux sera lente (vitesse de montée des eaux correspondant à celle du niveau marin).

\section{Références bibliographiques}

IGIGABEL M., NEDELEC Y., FLOUEST N., BERENGER N., CHASSE P., BERNARD A., COSQUER E., TIBERI-WADIER A.-L. (sous presse). Étude des systèmes de protection contre les submersions marines. Méthodologie et études de cas issues du retour d'expérience Xynthia, Éditions du Cerema. 